\title{
Thiophene Metabolism by E. coli
}

\author{
Contract DE-FG22-87PC79912
}

\author{
David P. Clark \\ Department of Microbiology \\ Southern Illinois University \\ Carbondale, Illinois 62901
}

\section{DISCLAIMER}

\begin{abstract}
This report was prepared as an account of work sponsored by an agency of the United States Government. Neither the United States Government nor any agency thereof, nor any of their employees, makes any warranty, express or implied, or assumes any legal liability or responsibility for the accuracy, completeness, or usefulness of any information, apparatus, product, or process disclosed, or represents that its use would not infringe privately owned rights. Reference herein to any specific commercial product, process, or service by trade, name, trademark, manufacturer, or otherwise does not necessarily constitute or imply its endorsement, recommendation, or favoring by the United States Government or any agency thereof. The views and opinions of authors expressed herein do not necessarily state or reflect those of the United States Government or any agency thereof.
\end{abstract}

Final Technical Report

September 15, 1987-December 31, 1991 


\section{EXECUTIVE SUMMARY}

We have previously isolated mutants of Escherichia coli which show increased oxidation of heterocyclic furan and thiophene substrates. We have now found that strains carrying the thd $A$ mutation express a novel enzyme activity which oxidizes a variety of substrates containing a sulfone $\left(\mathrm{SO}_{2}\right)$ moiety. Both heterocyclic sulfones (e.g., tetramethylene sulfone) and simple aliphatic sulfones (e.g., ethyl sulfone) were oxidized. The thdA mutants were more resistant than wild-type strains to aromatic sulfone antibiotics such as dapsone. In contrast, they showed increased susceptibility to thiolutin, a cyclic antibiotic containing sulfur at the sulfide level of oxidation. Several new thdA mutant alleles were isolated by selecting for increased oxidation of various aliphatic sulfur compounds. These new thdA mutants showed similar sulfone oxidase activity and the same map location (at $10.7 \mathrm{~min}$ ) as the original thdA1 mutation. The constitutive fadR mutation was required for the phenotypic expression of thdA-mediated oxidation of sulfur compounds. However, the thdAdirected expression of sulfone oxidase activity was not fadR dependent. The thdC and thdD mutations probably protect against the toxicity of thiophene derivatives rather than conferring improved metabolic capability.

Some of the genes involved in thiophene oxidation were cloned into the multicopy vector pUC19. The plasmid pKA 10 carries a $3.8-\mathrm{kb}$ chromosomal fragment which encodes a previously undiscovered gene involved in thiophene oxidation. Three proteins with approximate molecular sizes of 48, 30, and $26 \mathrm{kDa}$ were overproduced by cells carrying pKA 10. Maxicell experiments and DNA sequence analysis indicated that the 48- and 26-kDa proteins are encoded by pKA 10, whereas the 30-kDa protein is chromosomally derived. A cassette specifying kanamycin resistance was inserted into various sites on pKA 10. An insertion which abolished the 48-kDa protein also abolished thiophene oxidation. Chromosomal integration 
of pKA 10: Kan allowed us to locate the chromosomal insert of pKA 10 at 84 min on the E. coligenetic map by transduction. Since no previously identified genes involved in thiophene metabolism are located in this region, we designated the gene for the 48-kDa protein as thdF. Sequencing of the 3.8-kb insert revealed an overlap of several hundred bases with the regulatory and structural regions of the tnaA gene, which is also located at $84 \mathrm{~min}$. The $26-k$ Da protein is probably truncated tnaA protein. An open reading frame corresponding to the $48-\mathrm{kDa}$ thd $F$ protein was located next to the tnaA gene, which encodes tryptophanase, but was transcribed in the opposite sense.

Construction of thdF-lac gene fusions demonstrated that tha $F$ is induced by aerobic conditions and shows increased expression as cells enter stationary phase. 


\section{BACKGROUND}

We had previously isolated multiple mutants of Escherichia coli which had gained the capacity to oxidize certain thiophene derivatives and their furan analogs. It turned out that mutations in three novel genes (thd $A$, thd $C$, and thdD) and one previously described gene $($ thd $B=f a d R$ ) were involved in thiophene oxidation. The thd $A$, thd $C$, and thdD mutations were mapped and the physiology of strains carrying these mutations was characterized. This initial work was published just prior to the start date for this project:

Abdulrashid N., and D. P. Clark. 1987. Isolation and genetic analysis of mutations allowing the degradation of furans and thiophenes by Escherichia coli. J Bacteriol 169 1267-1271.

The objective for this project was to continue the genetic analysis of the thiophene oxidation system, in particular:

1. characterization of the biochemical pathway for thiophene oxidation,

2. identification and mapping of any further genes involved in thiophene degradation,

3. analysis of how the thd genes are regulated, and

4. cloning and sequencing of at least some of the thd genes.

\section{PUBLICATIONS}

The majority of our results have been publisined as follows:

1. Clark, D. P., K. Y. Alam, N. Abdulrashid, and B. Klubek. 1988. Successive mutation of E. coli for improved thiophene degradation. Appl. Biochem. Biotechnol. 18 393-401. 
2. Alam, K. Y., M. J. Worland, and D.P. Clark. 1990. Analysis and cloning of genes involved in thiophene and furan oxidation by Escherichia coli. Appl. Biochem. Biotechnol. 24/25 843-855.

3. Juhl, M. J., and D. P. Clark. 1990. Thiophene degrading mutants of E. coli possess sulfone oxidase activity and show altered resistance to sulfur containing antibiotics. Appl. Environ. Microbiol. 56 3179-3185.

4. Alam, K. Y., ani D. P. Clark. 1991. Molecular cloning and sequence of the thdF gene, which is involved in thiophene and furan oxidation by Escherichia coli. J. Bacteriol. 173 6018-6024.

[A copy of paper \#4 is attached; the others have been provided with previous quarterly reports.]

\section{SUMMARY OF PUBLISHED WORK}

Our previous work resulted in the isolation of a series of mutants of $E$. coli with increasing ability to oxidize furan and thiophene derivatives. Mutations in three novel genes were involved, thdA, thdC, and thdD together with mutations in fadR ("thd $B$ ") and ato $C$. Of these thdA gave the biggest observable effect and we have therefore focused on this gene in our more recent work.

We thought it possible that thdA coded for a non-specific cytochrome $P 450$ type oxidation system, however the characteristic spectral peak at $450 \mathrm{~nm}$ in the presence of carbon monoxide was not observed in our thdA strains. Enzyme assays have suggested that thaA may code for a methylene blue linked oxygenase. The methylene blue linked reactions observed appear to fall into two classes. Class 1 reactions were observed with furoic acid, thiophene methanol and related substrates. These reactions were found in both wild type and thd $A$ mutants and do not appear to consume molecular oxygen. In contrast, Class 11 reactions occurred 
with tetramethylerie sulfone and sulfoxide and cyclohexanone. Class II reactions seem to be much greater in thdA strains than in wild type bacteria. Furthermore, Class $\|$ reactions also work when methylene blue is replaced by molecular oxygen and the reaction is run in an oxygen electrode chamber.

We have surveyed the effect of several sulfur containing antibiotics against the thiophene oxidizing mutant NAR 11 thdA, and some related strains. Strains carrying the thdA mutation were more sensitive to thiolutin, thiamphenicol and ampicillin however they showed enhanced resistance to aromatic sulfone antibiotics such as daspsone or thiazolsulfone. In contrast introduction of the thdC mutation greatly increased the sensitivity to the aromatic sulfones. Since the thdA mutation specifies oxidation of sulfones, it is possible that thdA mutants protect themselves against sulfone antibiotics by increased degradation of these agents.

Mutants resistant to the sulfone derivative, thiamphenicol were isolated. However, none were specific for this sulfur compound as they all shared cross resistance to the parent antibiotic chloramphenicol.

We isolated several new thdA mutants which mapped at the same location on the $E$. coli chromosome as the original thdA1 mutation of NAR11. These mutants were isolated for increased oxidation of ethane sulfonic acid or betamercaptoethanol. However, when these rinutations were moved into DC625, they showed the same ability to oxidize furans and thiophenes as the original NAR 11 thdA1 mutant.

The thdA1 and thdF genes were cloned onto the plasmids pKA15 (thdA1) and pKA 10 (thdF). The DNA sequence of the chromosomal insert of pKA 15, carrying the thdA1 mutant version of the thdA gene from strain NAR30 has been completely sequenced.

The chromosomal insertion of plasmid pKA 10 was also fully sequenced. This 3612 base pair sequence includes an open reading frame of approximately $2200 \mathrm{bp}$. 
This is the previously designated thdF gene which encodes a protein of approximately 48 kilodaltons. The insertion of a kanamycin resistance cassette which abolished production of the thdF protein was found to lie within this open reading frame. A homology search through Gene-Bank suggested that the chromosomal DNA segment encoding thdF lies close to and overlaps the thaA (tryptophanase gene) at $83.5 \mathrm{~min}$ on the E. coli chromosomal map. This confirmed our own genetic mapping of thdF and suggested a possible relationship to genes involved in aromatic aminoacid degradation.

We found that our thiophene degrading mutants of $E$. coli oxidized naphthalene sulfonic acids slowly and were poisoned by indole. One interpretation of these results is that the thd system, including thdF has reactivated an obsolete metabolic pathway for degradation of indole and related bicyclic ring compounds.

\section{RECENT UNPUBLISHED RESULTS}

Our published work on the characterization of the thd metabolic system for the oxidation of furans and thiophenes has been discussed above. We have identified several genes involved in the thd system of which two are of importance here: thd $A$ the putative repressor gene and $t h d F$, one of the structural/metabolic genes which is under thdA control.

Since cloning and sequencing the thdF structural gene together with its regulatory region (4) we have constructed thdF-lac $Z$ operon fusions (Bunch and Clark, unpublished). These were constructed by using a PCR approach. Primers corresponding to sequences located close to the start of translation and approximately halfway through the tnaA---thdF intergenic region were used to generate the thdF regulatory region as a PCR fragment (Figure 1). This was then cloned into an operon fusion vector in front of the lacZ gene. The PCR primers each included an artificial restriction site to facilitate the cloning. The thdF regulatory 


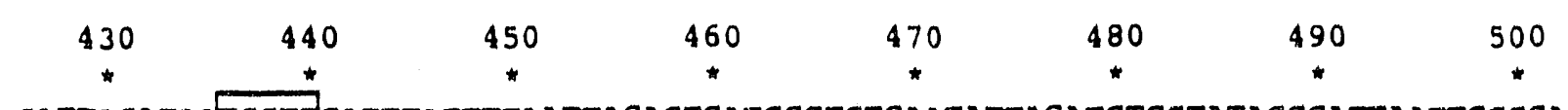

CATTACATANCCTMCATTATTMATTACAGTGATCCCTGTGATATTACATCTGCTATACCGATIAATCGCCAGAT Met<etnaA RBS

$\begin{array}{cccccccc}510 & 520 & 530 & 540 & 550 & 560 & 570 & 580 \\ \star & \star & \star & \star & \star & \star & \star\end{array}$

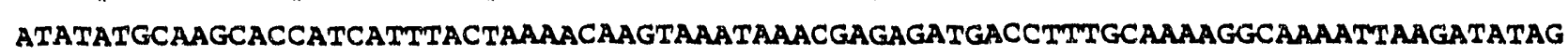

$\begin{array}{cccccccc}590 & 600 & 610 & 620 & 630 & 640 & 650 & 660 \\ * & * & * & * & * & * & * & *\end{array}$

CAAACAAATAGAACACATTGAATCAATCGGTTGGCTCTGGTGATGGCTACAGAAGGGCAAATCAAGGGCGGGATCGAC
ProArgH isaspVal

$\begin{array}{ccccccccc}670 & 680 & 690 & 700 & 710 & 720 & 730 & 740\end{array}$

AATWTATTGCAATATIGAACCATITGAGGTCAACACATATATGTAAGATATTCATAATGCACWTACCTCGCAAGAC IleLysAs nAs oI leAs nP heTrpLys SerThrValCys I leBisLeu I leAsnMet.

$\begin{array}{cccccccc}750 & 760 & 770 & 780 & 790 & 800 & 810 & 820 \\ \star & \star & * & * & * & * & \text { CRP } & *\end{array}$

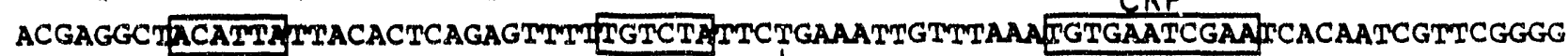
$-10$

$-35$

PCR Primer A

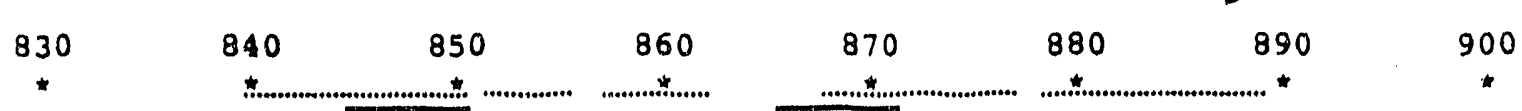

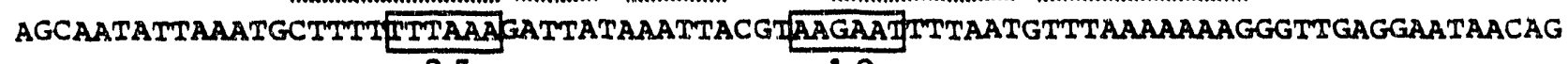
$-35 \quad-10$

$\begin{array}{cccccccc}910 & 920 & 930 & 940 & 950 & 960 & 970 & 980\end{array}$

GAGCGGTAACAATGAAGCGAAGCGCATCTGACAGTCAGAATGCGGCTGTAT̈GCGCGGTTMCTTGATGTCTICGACCTCT Gear Box

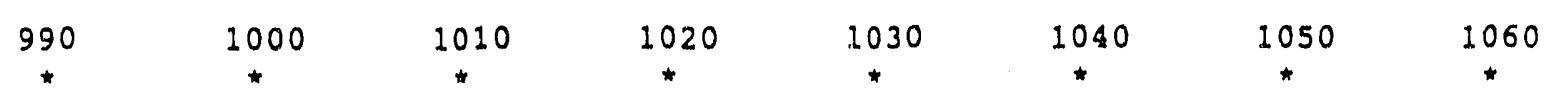

TTTACGCGGGGTCGTCCAGCAGACTTCATIPAAGGGCCACTAAATGGATTGCAAGACGACACGGTCTGCGTTGAGAAG

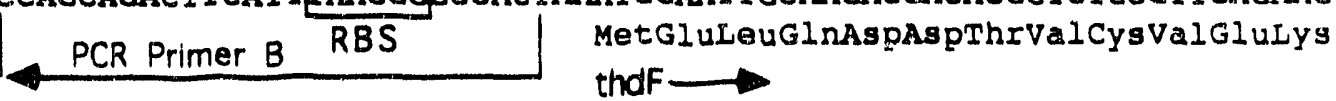

Figure 1: Intergenic region between tnaA and thdF. The sequence shown is the coding strand for thd $F$ but the complementary strand for tnaA and tnaC. Coordinates are according to Alam and Clark (2).
RBS $=$ ribosome binding sites
CRP = crp binding site for tnaA
tnaC = leader peptide for tnaA operon
Gearbox $=$ stationary phase consensus sequence
840-889 $=$ putative stem and loop; inverted repeats are
85-1027 = PCR fragment used to generate thdF-lacZ operon
fusions
$754-781=$ promoter for tnaA/tnaC
$845-872=$ computer predicted promoter for thdF 
region, so defined, includes the CRP-site used by both tnaA and thdF, a prominent stem and loop structure, a computer predicted promoter ( -35 and -10 sequences) and a stationary phase gearbox sequence (Figure 1). The original gene fusions were constructed on the plasmid vector pRS 414, however, we have also transferred the fusions to the chromosome, so as to assay them when present in single copy.

We have demonstrated that the thdF-lac $Z$ fusions are induced 10 to 15 -fold when cells enter stationary phase and that thdF-lac $Z$ is subject to moderate (2 to 4fold) catabolite repression by glucose. In addition to these effects we have found that there is almost no expression of thdF-lacZ under anaerobic conditions (even in stationary phase and in the absence of repression by glucose). Finally, the thdF-lac $Z$ fusion is subject to the thd regulatory system itself. Not surprisingly, the thdF-lac $Z$ fusion, when on a multicopy plasmid, expresses beta-galactosidase at a higher level than the single copy chromosomal fusions. The plasmid borne fusion also largely escapes from thdA mediated repression, presumably due to multiple copies of the promoter out numbering the available repressor molecules. However, aerobic regulation, catabolite repression and stationary phase induction are observed with the multicopy version of the fusion-presumably because the global regulator proteins responsible for these phenomena are present in sufficient numbers to titrate the multiple copies of the thdF promoter.

We have so far isolated several spontaneous mutants in which the thdF-lacz fusion is expressed anaerobically. These were selected directly by anaerobic growth with lactose as carbon source. A few of these showed increased sensitivity to toluidine blue, suggestive of arcA. However, the majority showed no such alteration. Genetic analysis of these mutarits is still in progress. 

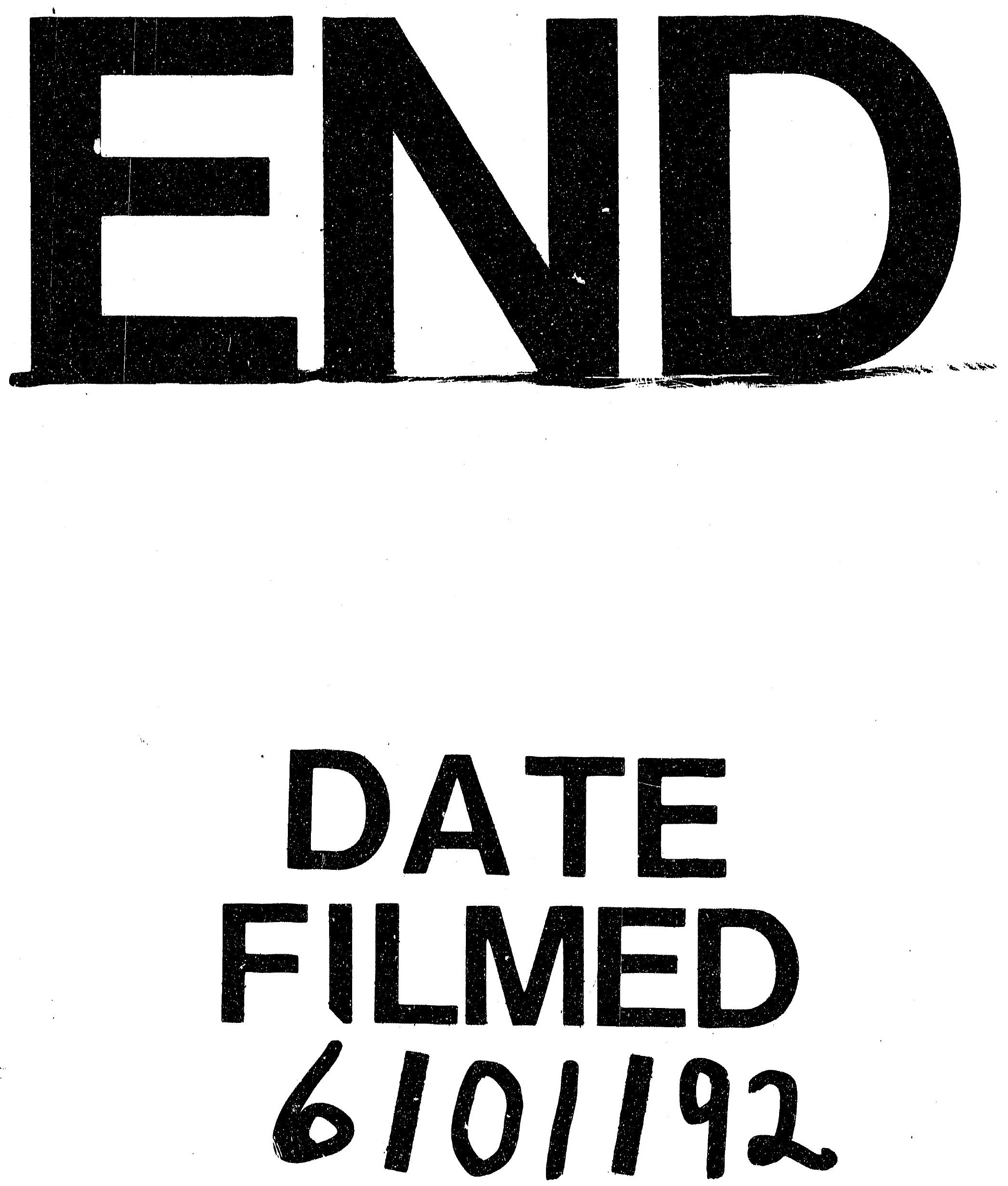
